\title{
Kartierung von Höhengrenzen zwischen Mt. Blanc und Gotthard-Massiv mit Skylab-EREP-Aufnahmen
}

\section{Aufnahmematerial}

Für die vorliegenden Untersuchungen wurden sämtliche photographischen Produkte von Skylab-3, der 2. bemannten Skylab-Mission vom 28. Juli bis 25. September 1973 entsprechend, $\mathrm{Pa}$ 21, vom 11. September 1973 , aufgenommen zwischen 15.26 Uhr und 15.27 Uhr, verwendet. Die nachfolgende Zusammenstellung zeigt die benützten Filme (alles Kodak-Materialien) und die genauen Bilddaten:

$S$ 190-A MPC (multispectral photographic camera): sechs Kameras mit Objektiven 1:2,8/152; Format $7 \times 7$ $\mathrm{cm}$, Originalmaßstab ca. 1:2800000 (eine Aufnahme bedeckt ca. $25600 \mathrm{~km}^{2}$ ):

\begin{tabular}{llll}
\hline & $\begin{array}{l}\text { Spektralbereich } \\
\mu \mathrm{m}\end{array}$ & Filmtyp & $\begin{array}{l}\text { Theoretische } \\
\text { Bodenauflösung in m }\end{array}$ \\
\hline Schwarzweiß-Filme & $0,5-0,6$ & PAN-X SO-022 & $40-46$ \\
& $0,6-0,7$ & PAN-X SO-022 & $30-38$ \\
& $0,7-0,8$ & IR EK-2424 & $73-79$ \\
Farbfilm & $0,8-0,9$ & IR EK 2424 & $73-79$ \\
Infrarot-Farbfilm & $0,4-0,7$ & High-Res. SO-356 & $40-46$ \\
& $0,5-0,88$ & Aerochrome IR & $73-79$ \\
\hline
\end{tabular}

$S$ 190-B ETC (earth terrain camera): Objektiv 1:4/457; Format $13 \times 13 \mathrm{~cm}$, Originalmaßstab ca. 1: 900000 (eine Aufnahme bedeckt ca. $10700 \mathrm{~km}^{2}$ ); Normal-Farbfilm Typ Aerialcolor High-Res. SO-242, ohne Filter, theoretische Bodenauflösung ca. $17 \mathrm{~m}$.

Die atmosphärischen Verhältnisse waren am Aufnahmetag infolge starker Bewölkung (siehe Titelphoto) recht ungünstig. Eine Wiederholung der Aufnahme für vergleichende Untersuchungen fand zwar am

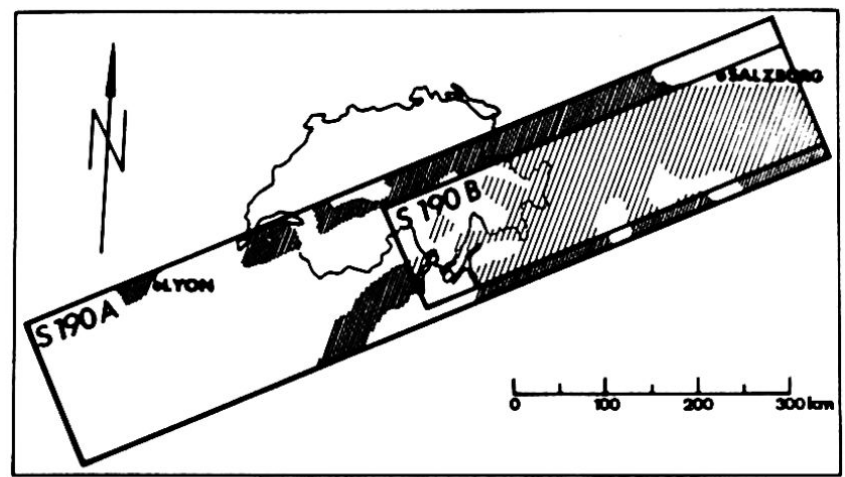

Fig. 1 Skylab Paß 21 vom 11. September 1973: Überdeckungsbereich der photographischen Aufnahmesysteme
19. September 1973 auf einer um ca. $15 \mathrm{~km}$ gegen $\mathrm{N}$ verschobenen Umlaufbahn statt, allerdings bei ca. 95\% Wolkenbedeckung. Weitere Überdeckungen waren mehrfach während der 4. Skylab-Mission (16. November 1973 bis 8. Februar 1974) vorgesehen, mußten aber aus technischen Gründen immer wieder verschoben werden und fielen schlußendlich ganz aus.

Gleichwohl läßt sich das vorhandene Material in vielfältiger Weise ausschöpfen. In der vorliegenden Arbeit sollen insbesondere methodische und regionale Aspekte im Zusammenhang mit der Kartierung von Höhengrenzen im alpinen Raum untersucht werden, wie:

- Zustandserfassung und Kartierung der temporären Schneegrenze und der Obergrenze des Pflanzenwachstums über größere Gebiete hinweg

- Regionaler Vergleich der Grenzverläufe

- Vergleichende Betrachtung der Grenzverläufe unter speziellen Verhältnissen (z. B. Schneegrenze auf Gletschern)

Prof. Harold Haefner und Urs Geiser, Assistent, Geographisches Institut der Universität Zürich, Blümlisalpstraße 10, 8006 Zürich 
- Vergleich der Aufnahmesysteme, speziell hinsichtlich Kartierungsgenauigkeit

- Günstigster Kartierungsmaßstab (Kartenunterlage) Bei der Interpretation wurde bewußt auf moderne Bildverarbeitung oder digitale Klassifikationsverfahren verzichtet und mit den konventionellen Methoden der Photointerpretation unter Anwendung einfacher, allgemein zugänglicher Hilfsmittel (Stereoskop, Stereolupe, Projektor usw.) visuell gearbeitet. Die Auswahl des Untersuchungsgebietes ergab sich aus der Umlaufbahn (Fig. 1) und den Bewölkungsverhältnissen.

\section{Kartierung der temporären Schneegrenze mit S 190-A}

Für die Festlegung einer Grenze zwischen schneebedeckten und schneefreien Gebieten wie Gletschereis, Alpweiden, Wald, Fels, Schutthalden usw. in verschiedenen Expositionen, Hangneigungen und in Sonnenund Schattenlage, wurden vor allem folgende Abbildungsmerkmale herangezogen:

- Eindeutiger Kontrast der hellen Schneedecke zur dunkleren Umgebung (Interpretationskriterium: Farbton)

- Großflächigere Form der Schneedecke mit feinziseliertem Rand im Gegensatz zur kleinförmig gegliederten Umgebung (formales Interpretationskriterium)

Diese Merkmale sind allerdings oft lokal gestört und erschwerten dadurch eine Interpretation:

- Wolken können in gleich hellen Tönen wie Schnee abgebildet werden (Unterscheidungsmerkmal: Form und Randausbildung; "gewundene, längliche» Form der Schneedecke mit dendritischer Randausbildung gegenüber der «massigen» Form der Wolken mit "geradliniger, faserigen» Abgrenzung)

- Helligkeitsunterschiede zwischen Hängen in Sonnen- und Schattenlagen

- Bei abschmelzendem Schnee entsteht keine scharfe Grenzlinie sondern eine Übergangszone (Generalisierungsproblem)

Diese Effekte erschwerten eine exakte Grenzziehung stark. Die Interpretation wurde deshalb mit Hilfe einer Stereolupe (16fache Vergrößerung) unter ständigem Vergleichen von Farb- und Falschfarben-Transparentpositiven sowie der Schwarzweiß-Aufnahmen durchgeführt.
Der große Vorteil der Satellitenaufnahmen liegt darin, daß sie im Gegensatz zu großmaßstäbigen Luftbildern den Schneegrenzbereich weniger als Saum, sondern eher als Linie wiedergeben (geringere Bodenauflösung), also eine gewisse Generalisierung vorwegnehmen.

Allgemein wurde auf dem Normal-Farbfilm interpretiert, während sich der Infrarot-Farbfilm besonders für die Abgrenzung der Schneedecke auf dem Gletscher eignet, da er eine bessere farbliche Trennung zwischen den beiden Objekten aufweist. Der Grund hierfür liegt im unterschiedlichen Reflexionsverhalten von Schnee und Eis (Fig. 2). Wie ersichtlich, verlaufen die beiden Reflexionskurven im sichtbaren Bereich des Spek-

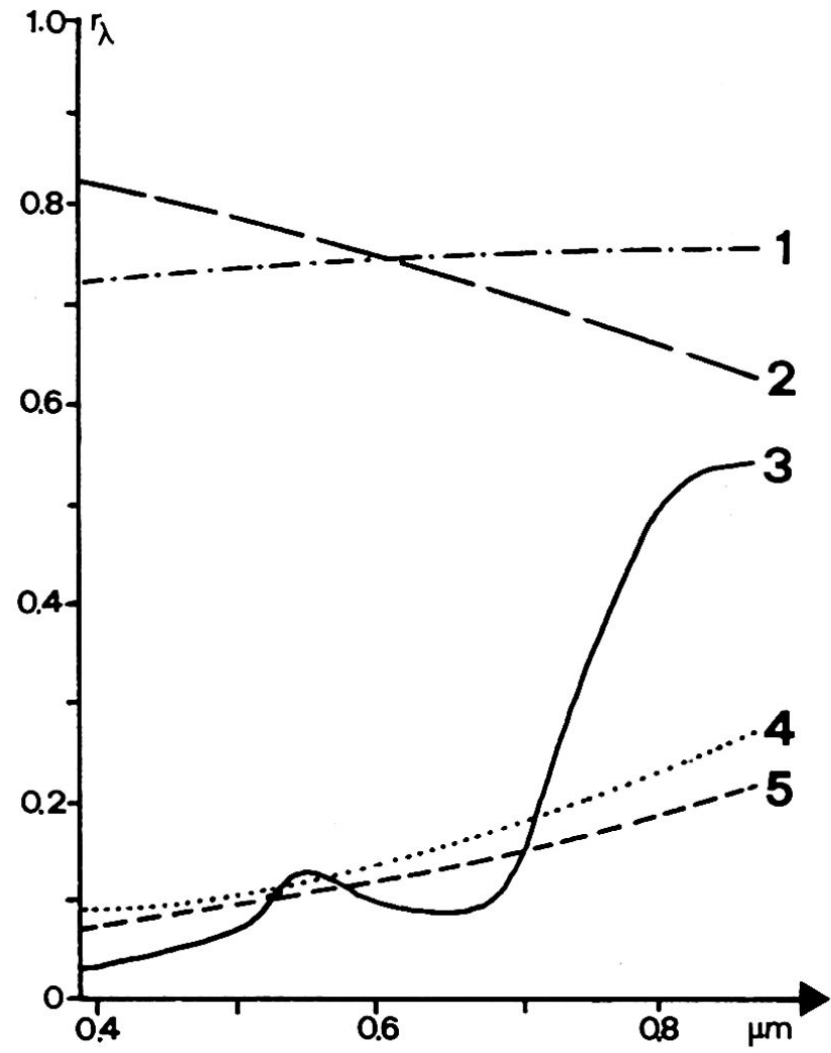

Fig. 2 Spektrale Reflexion verschiedener Oberflächeneinheiten im sichtbaren Bereich und nahen Infrarot
1 - Eis
4 - tote Vegetation
2 - Schnee
$5=$ Gestein
3 - lebende Vegetation

(nach KONDRATYEV et al, 1974, leicht ergänzt) 
trums sehr ähnlich, während sie im nahen Infrarot auseinanderstreben und damit eine bessere farbliche Trennung im Falschfarbenphoto belegen. Eine Trennung zwischen Schnee und Vegetation oder Gestein zeigt hingegen im sichtbaren Bereich die größeren Unterschiede.

Die Übertragung der Schneegrenze in die Karte erfolgte mit Hilfe eines Projektionsapparates. Das interpretierte EREP-Transparent-Farbbild wurde direkt in die Landeskarte 1:200000 projiziert und anhand eindeutig fixierbarer Punkte wie Flußmündungen, Staumauern und Bergspitzen eingepaßt. Es zeigte sich, daß mit entsprechendem Zeitaufwand genügend $\mathrm{Paßpunkte}$ für eine genaue Korrelation gefunden werden konnten. Gegen den Bildrand traten kleinere Verzerrungen auf, die sich aber durch Verkippen der Projektionsfläche beseitigen ließen. Die Bilddeformationen sind recht gering, da zufolge der großen Flughöhe und des kleinen Öffnungswinkels des Objektivs auch im alpinen Relief nur geringe Abweichungen resultieren.

Die LK 1: 200000 erwies sich nach verschiedenen Versuchen als die geeignetste Kartenunterlage für eine übersichtliche Darstellung. Die Photos könnten zwar sogar auf die LK 1: 100000 vergrößert werden, doch würden die Ergebnisse auf zu viele Kartenblätter verteilt.

\section{Kartierung der Obergrenze des Pflanzenwachs- tums mit S 190-A}

Wie aus Fig. 2 ersichtlich, reflektiert lebende Vegetation im nahen Infrarot wesentlich stärker als tote. Deshalb wird im Infrarot-Farbfilm wachsende Vegetation rot, tote dagegen in gelblich-bräunlichen Tönen widergegeben.

Eine erste visuelle Durchsicht der Aufnahmen ergab, daß im Aufnahmezeitpunkt das Wachstum in die Höhe bereits ausgesetzt hatte und im Bereich der Alpweiden ein breites Band toter Vegetation zwischen Schneeund Wuchsgrenze bestand. Letztere lag aber noch über der Waldgrenze. Die Voraussetzungen für eine Kartierung waren somit günstig, denn innerhalb des Nadelwaldes wäre der Verlauf der Wachstumsgrenze viel schwieriger festzulegen gewesen.

Die Obergrenze des Pflanzenwachstums ließ sich also anhand der Rotfärbung eindeutig interpretieren. Es wurden die gleichen methodischen Verfahren ange- wendet wie für die Schneekartierung und die Ergebnisse in entsprechender Weise in die LK 1:200000 übertragen.

Die Obergrenze der Vegetation konnte dagegen mit Hilfe der vorliegenden Aufnahmen nicht festgelegt werden, da sich die Vegetationsdecke gegen oben allmählich immer mehr auflöst. Zudem war sie im Aufnahmezeitpunkt teilweise bereits von Schnee überdeckt. Die Reflexionskurven zwischen toter Vegetation und Gestein zeigen ebenfalls minimale Unterschiede (Fig. 2). Gleichwohl hebt sich das Gestein mit seinem mehr grau-bläulichen Farbton deutlich vom Band der toten Vegetation ab, allerdings mit einer fließenden Übergangszone. Zur Kartierung der Obergrenze der Vegetation wäre eine Aufnahme im Spätsommer (max. Vegetationsentwicklung) notwendig.

\section{Ergebnisse der S 190-A Kartierungen}

Fig. 3 zeigt einen kleinen Ausschnitt der Kartierungen, die für das gesamte Untersuchungsgebiet durchgeführt wurden. Für vergleichende regionale Betrachtungen konnte die Lage der Höhengrenzen leicht aus der Karte entnommen und in tabellarischer Form nach verschiedenen Einteilungskriterien zusammengestellt werden. Dabei ließen sich sowohl exakte Werte für eine bestimmte Lokalität als auch durchschnittliche Beträge für einen ganzen Talhang ermitteln. Die LK 1:200 000 mit einer Aequidistanz von $200 \mathrm{~m}$ erlaubt dabei eine Interpolation der Höhenlage mit einer Genauigkeit von ca. $\pm 50 \mathrm{~m}$.

Die regionalen und expositionellen Unterschiede im Verlauf der temporären Schneegrenze wie der Pflanzenwachstumsgrenze sind beachtlich. Besonders interessant ist die Lage der Schneegrenze auf den Gletschern. Vom Datum her gesehen entspricht sie annähernd der maximalen Position im Spätsommer und dürfte damit wenigstens annähernd Hinweise auf den Verlauf der klimatischen Schneegrenze geben. In der nachstehenden Tabelle ist die Lage der temporären Schneegrenze auf den Gletschern des nördlichen Wallis (Lötschental-Furka) nach Exposition getrennt zusammengestellt. Das Pflanzenwachstum in den oberen Regionen der alpinen Stufe hat zu diesem eher frühen Datum bereits ausgesetzt. Auch hier lassen sich aufschlußreiche phänologische Differenzierungen erkennen. 
Fig. 3 Höhengrenzen, kartiert mit S 190-A im Gebiet Lötschental-Großer Aletschgletscher auf LK 1: 200000

Fig. 4 Temporäre Schneegrenze im Gebiet FinsteraarhornUnteraargletscher, kartiert auf LK 1: 100000

(Kartenausschnitte reproduziert mit Bewilligung der Eidg.

Landestopographie)
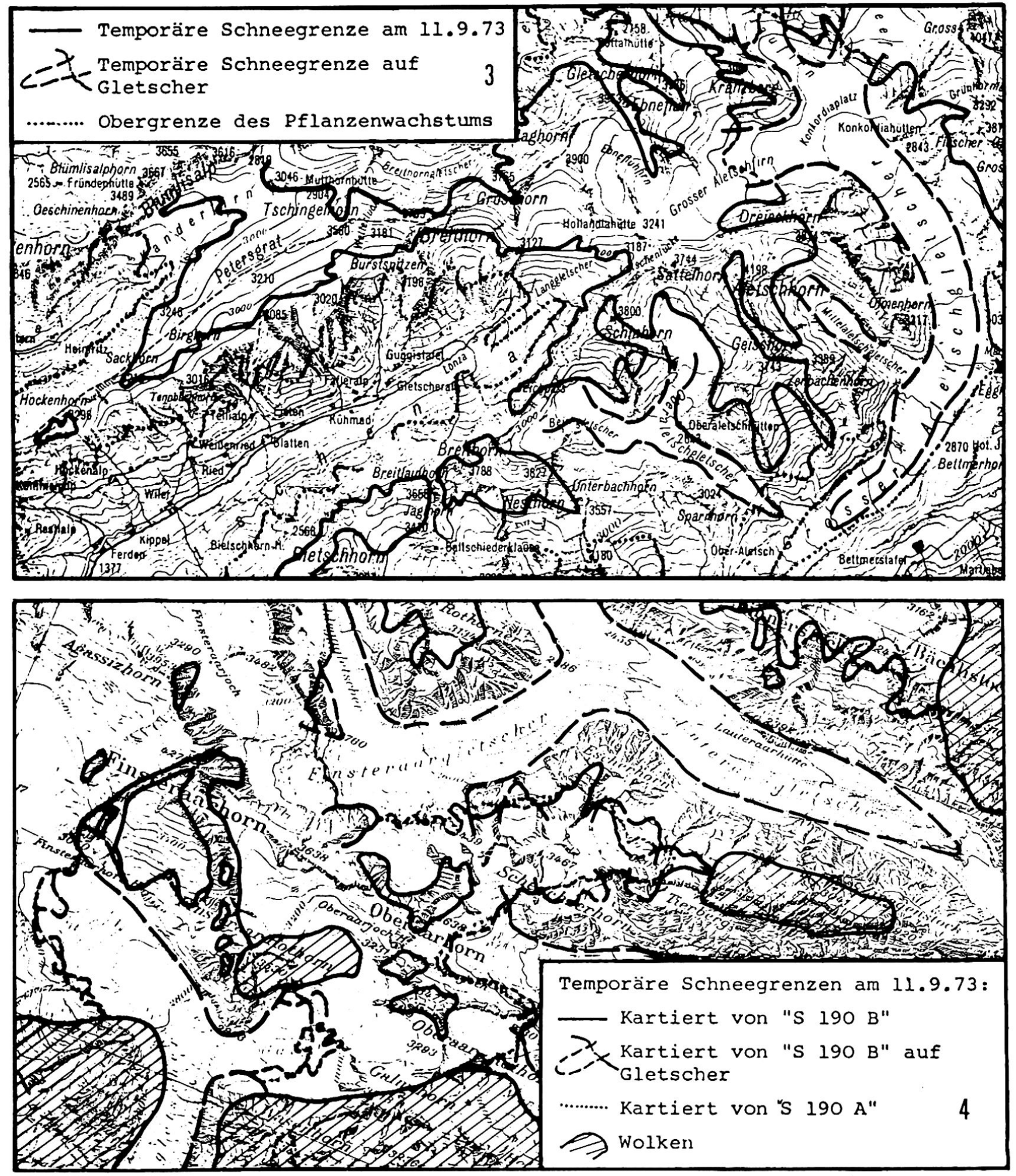
Durchschnittliche Lage der temporären Schneegrenze am 11. September 1973 auf den Gletschern des nördlichen Wallis

\begin{tabular}{lll}
\hline Exposition. & $\begin{array}{l}\text { Anzahl } \\
\text { Gletscher }\end{array}$ & $\begin{array}{l}\text { durchschnittl. Lage der } \\
\text { temp. Schneegrenze }\end{array}$ \\
\hline $\mathrm{N}$ & 2 & $2750 \mathrm{~m}$ \\
$\mathrm{E}$ & 5 & $2970 \mathrm{~m}$ \\
$\mathrm{~W}$ & 1 & $3000 \mathrm{~m}$ \\
$\mathrm{~S}$ & 8 & $2890 \mathrm{~m}$ \\
\hline
\end{tabular}

\section{Kartierung der temporären Schneegrenze mit S-190-B}

Die Earth-Terrain-Kamera mit einem Auflösungsvermögen von ca. $10 \mathrm{~m}$ gestattet eine noch genauere Abgrenzung. Vergleiche können nur über den Verlauf der Schneegrenze (da nur Normal-Farbfilm) vorgenommen werden. Infolge der größeren Brennweite wird ein wesentlich kleinerer Geländestreifen erfaßt, wobei die Aufnahmen aus bekannten Gründen erst im östlichen Wallis und Tessin einsetzen (Fig. 1). Als effektiv vergleichbares Gebiet bleibt darum vor allem die Grimsel-Region (Fig. 4).

Das methodische Vorgehen entsprach demjenigen, das beim S 190-A angewandt wurde. Die Interpretation wurde von der gleichen Person vorgenommen, aber mit einer Zeitdifferenz von ca. zwei Monaten, so daß keine "vorgefaßte» Darstellung mehr erfolgen konnte. Als Kartierungsgrundlage wurde diesmal entsprechend dem größeren Bildmaßstab die LK 1:100000 gewählt. Die Aequidistanz von $50 \mathrm{~m}$ ermöglicht eine Lokalisierung der Höhengrenze auf ca. $\pm 20 \mathrm{~m}$.

\section{SchluBfolgerungen}

Hochauflösende Satellitenphotos ermöglichen infolge ihres synoptischển Blickfeldes erstmals eine präzise Kartierung von Höhengrenzen über größere Gebiete hinweg unter absolut identischen Voraussetzungen. Daraus ergeben sich die Grundlagen für echte vergleichende Untersuchungen verschiedener Regionen mit guter Genauigkeit und großem Detailierungsgrad.

Zeitliche Veränderungen würden sich mit Hilfe von wiederholten Überdeckungen festhalten lassen, und daraus könnten Kenntnisse über die dynamischen Veränderungen im jahreszeitlichen Rhythmus gewonnen werden.

\section{Literaturverzeichnis}

BARNES, JAMES C. Und BOWLEY, CLINTON J.: Handbook of Techniques for Satellite Snow Mapping. ERT Document No 0407-A for NASA, Concord, Mass. 1974.

KONDRATYEV, K. YA. et AL: Results of Spectrometric Measurements of Natural Formations from the Spacecraft «Soyuz-9». in: Remote Sensing of Environment, No 1, S. 15-27, New York 1974.

SKYLAB Earth Resources Data Catalog: L. B. Johnson Space Center, Houston, Texas 1974. 